stationary processes. The asymptotic behavior of quadratic estimates of covariances of linear processes is given. The chapter concludes with a discussion of some results on the estimation of parameters of finite parameter schemes.

Chapter three (Estimation of the Spectral Density and Distribution Functions) considers the asymptotic behavior of estimates of the spectral density from the point of view of covariance structure. The fact that the periodogram is not a consistent estimate is noted and weighted averages of the periodogram are proposed. An estimate of the variance of such estimates in terms of the weight function is obtained for large sample size. Various weight functions are discussed in detail.

The fourth chapter (Hypothesis Testing and Confidence Intervals) begins with a discussion of a classic test for a possible periodic component when the residuals are independent. The question of plausible modifications of the test for a more general stationary residual is considered. A test for independence against a simple Markovian alternative is given. Discussion of tests of goodness of fit follow and finally a discussion of a technique for getting a confidence region for the spectral distribution function.

Most of the discussion up to this point assumes zero mean. The final chapter (Processes Containing a Deterministic Component) first discusses the estimation of regression coefficients. It is shown that the least squares estimates are asymptotically efficient in the class of linear unbiased estimates for a large class of regressions. There are suggested modifications of some of the techniques presented in the previous chapters to take care of the case of nonzero mean.

Much of the discussion is of a heuristic character but this is inevitable in a book of this size. There is a remarkably broad coverage of some of the recent results in time series analysis, particularly those on spectral analysis. Altogether this monograph is a welcome addition to the growing literature on time series analysis.

\title{
M. Rosenblatt
}

Integral quadratic forms. By G. L. Watson. Cambridge Tracts in Mathematics and Mathematical Physics, No. 51. Cambridge, 1960. $12+143$ pp. $\$ 5.00$.

This book is about classes, genera, and spinor genera of quadratic forms.

We recall that a rational quadratic form $g$ is said to be in the same class as a given form $f$ if there is an integral substitution of determinant \pm 1 which carries $f$ to $g$; the set of such $g$ is called the class 
cls $f$ of $f$. A rational quadratic form $g$ is in the same genus as $f$ if there is an integral $p$-adic substitution of unit determinant at each finite prime $p$, and a real substitution at infinity, all of which carry $f$ to $g$; the set of these $g$ is called the genus gen $f$ of $f$. It is important in the arithmetic theory of quadratic forms, as well as in applications, to have a good description of cls $f$. Now there are well-known characterizations of the genus, and these provide some information about the class since in general cls $f \subseteq$ gen $f$. However, a single genus will often consist of several classes. This fact has resulted in an extensive literature on the relationship between a genus and its classes; of particular interest to the author's point of view is the result of Meyer (1891) which gives conditions under which cls $f=$ gen $f$. No satisfactory proof of Meyer's result was known until a few years ago with the introduction of the spinor genus. The spinor genus spn $f$ is a set of forms equivalent to $f$ under a new relation to be described in a moment; it will satisfy cls $f \subseteq$ spn $f \subseteq$ gen $f$. Two things have to be done with spn $f$ once it has been defined: it must be compared with cls $f$ and it must be compared with gen $f$. First one shows that cls $f=\operatorname{spn} f$ for an indefinite form in at least three variables; then one gives a group theoretic description of the spinor genera in a genus, in particular one obtains a good way of counting them. These are the main steps in the theory as it has developed during the last ten years. As an example one obtains conditions under which cls $f=\operatorname{gen} f$.

The spinor genus is due to Eichler. His early results (1952) established the theory over the rational field and also, in certain special cases, over a number field. Kneser (1956) extended this to number fields in general. At about the same time Watson obtained Eichler's results by elementary methods over the rational field.

We can get a good idea of the two different approaches to this subject from the respective definitions of the spinor genus. In the first instance (this is the EichlerKneser approach) it is necessary to introduce certain concepts right from the start. These consist of a vector space $V$ provided with a quadratic form over an algebraic number field, a lattice $L$ in $V$, the $\mathbf{p}$-ifications $V_{\mathbf{p}}$ and $L_{\mathbf{p}}$, and the rotations and spinor rotations of $V$ and $V_{\mathbf{p}}$. One then says that a lattice $K$ is in spn $L$ if $L$ can be rotated onto some lattice $J$, and if each $J_{\mathbf{p}}$ can then be spinorially rotated onto $K_{\mathbf{p}}$.

In the second approach (this is the Watson approach) let $f^{R}$ denote the quadratic form obtained from $f$ by means of the substitution of new variables through the rational matrix $R$. Then $g$ is said to be in $\operatorname{spn} f$ if there is an $R$ such that

$$
g=f^{R} \text {, det } R= \pm 1 \text {, g.c.d. }(\operatorname{den} R, d)=1 \text {, norm } R=1 \text {. }
$$

Here den $R$ and norm $R$ are obtained from the invariant factors of $R$ in a certain way, and $d$ is the discriminant of $f$.

Needless to say, it is possible to reconcile the two points of view. 
The purpose of the book under review is to arrive at the theory of the spinor genus by elementary methods, i.e. using matrix calculus and assuming some knowledge of elementary number theory. Whatever is needed from the arithmetic theory of quadratic forms is defined and proved. The development takes place in three main parts: local and global field equivalence, integral $p$-adic equivalence and the genus, and finally the spinor genus.

Chapters 1-3 give the Hasse-Minkowski theory of fractional equivalence. The fundamental result that a global form represents 0 if and only if it does so locally everywhere is proved by reduction theory and Dirichlet's theorem on primes in an arithmetic progression.

Chapters 4-5 discuss local integral theory and the genus. Included is an important theorem on the integers represented by an indefinite form in at least four variables.

Chapters $6-8$ do the spinor genus. The difficult part here is to establish the relations between cls $f$, spn $f$, and gen $f$. Much time is also spent on rounding out the picture with additional results, some of them new.

The book suffers from an exasperating conceptual deficiency. Too often a formula or device is used to circumvent the introduction of an idea. Surely there is no longer any need to shy away from groups and vector spaces. And in a field that is so intimately concerned with questions of linearity, is it right to do so? Isn't it really better, and indeed simpler, to use $p$-adic numbers instead of families of congruences? We can sympathize with the author's efforts to keep out superfluous structure, but the criterion used in doing so should be conceptual, not just logical, necessity.

However, these are small matters. The important thing is that the author has contributed an ordered account of significant results in a field with a long history and a totally inadequate literature. The book will be read by people interested in quadratic forms, and it should provide an accessible reference for those who are interested in the applications.

O. T. O'MEARA

Fondements de la topologie générale. By Ảkos Császár. Akadémiai Kiadó, Budapest, 1960. 231 pp. $\$ 6.00$.

The author's goal is to treat uniform, proximity, and topological spaces from a common viewpoint. He accomplishes this by developing a very general theory of "syntopogenic structures" in which uniformities, proximities, and topologies emerge as particular cases. The idea is simple and interesting. A syntopogenic structure on a set $E$ is a family of (partial) ordering relations on $P(E)$ satisfying certain natural conditions. The family defining a topology, for example, 